\title{
Observer pitch and roll influence: The rod and frame illusion
}

\author{
JENNIFER E. CORBETT and JAMES T. ENNS \\ University of British Columbia, Vancouver, British Columbia, Canada
}

\begin{abstract}
Observers misperceive the orientation of a vertical rod when it is viewed in the context of a tilted frame (the rod and frame illusion, or RFI). The pitch and roll of the surrounding surfaces have independent influences on this illusion (Nelson \& Prinzmetal, 2003). Experiment 1 measured the RFI when the pitch and roll of the floor that supported the observer was varied, and the observer was either seated in a chair or standing upright. There were additive influences of pitch and roll on the RFI of seated but not standing observers. Experiments 2 and 3 decoupled body roll and head roll in order to isolate the vestibular and proprioceptive contributions to these effects. The results are interpreted in support of a hierarchy of influence on the RFI: Visual input is given top priority, followed by vestibular input, and then proprioceptive input.
\end{abstract}

This study examines how visual, vestibular, and proprioceptive sensory mechanisms combine to influence the perception of upright. Carnival funhouses and roadside attractions that distort our sense of upright give us firsthand experience in how these sensory systems interact. For example, inside a tilted "mystery cabin," people may appear to change in size as they move from one location to another, objects may appear to balance at impossible angles, and balls may seem to roll uphill (Shimamura \& Prinzmetal, 1999). Previous researchers have used the rod and frame illusion (RFI) in the laboratory to demonstrate our heavy reliance on vision in these types of circumstances. In the simplest type of experiment, surrounding a vertical rod with a tilted frame causes observers to misperceive the orientation of the rod in the direction of the frame (Asch \& Witkin, 1948a). The RFI is even stronger when observers are rolled to one side, as when they are inside a mystery cabin (Asch \& Witkin, 1948b), suggesting that observers rely even more on vision when their vestibular and proprioceptive senses are disturbed. A similar conclusion has been drawn with regard to induced disequilibrium and illusions such as the Zöllner, Poggendorff, and Ponzo illusions (Prinzmetal \& Beck, 2001).

The most is known about visual influences on the RFI. For example, a recent study compared the effects of changing an environment with regard to pitch (up and down slant of the viewed surfaces with respect to the viewer) and roll (side to side tilt of the viewed surfaces with respect to the viewer; Nelson \& Prinzmetal, 2003). The authors concluded that environmental pitch and roll contribute independently to the RFI. But pitch and roll of

This research was supported by a Natural Sciences and Engineering Research Council of Canada Discovery Grant to J.T.E. Correspondence concerning this article can be addressed to J. E. Corbett, Department of Psychology, University of British Columbia, Vancouver, BC, V6T 1Z4 Canada (e-mail: jecorbet@psych.ubc.ca). the viewed environment are not the only possible sources of disequilibrium in mystery cabins. Observers themselves are in an environment (their bodies) the surfaces of which are pitched (the body rotates about an imaginary axis running through the line of sight) and rolled (the body rotates about an imaginary axis that runs through the observer's ears). Yet little is known about these influences on the RFI, including whether observer pitch and roll have effects similar to those of visually perceived pitch and roll of the environment (Nelson \& Prinzmetal, 2003).

\section{EXPERIMENT 1}

We began by measuring the RFI in six conditions: three levels of observer roll $\left(0^{\circ}\right.$, or upright; $+20^{\circ}$ roll, congruent with the tilted frame; $-20^{\circ}$ roll, incongruent with the tilted frame) combined with two levels of observer pitch $\left(0^{\circ}\right.$, or upright; $-20^{\circ}$ pitch, backward with respect to the frame). In pilot experiments, we tested $0^{\circ}$ and $-20^{\circ}$ frame positions, in addition to a forward pitch condition. There was never any illusion in the $0^{\circ}$ condition, regardless of observer pitch or roll, pointing once again to the dominance of vision in this illusion. Furthermore, because the results for $-20^{\circ}$ and forward pitch were indistinguishable from those for $+20^{\circ}$ and backward pitch, respectively, we restricted ourselves to the latter conditions in order to maximize statistical power. Our choice of a $20^{\circ}$ magnitude for pitch and roll was based on two considerations: (1) The RFI is maximized when the visual frame is between $15^{\circ}$ and $22^{\circ}$ (Shimamura \& Prinzmetal, 1999), and (2) roll of the head or body results in a shift from an environmentally based sense of upright to an ego-based one when roll exceeds $60^{\circ}$ (Howard, 1982, 1986; Young, 1984).

In addition to examining observer pitch and roll, we addressed several other unresolved issues. For example, it is not clear whether there is a difference between rolling observers congruently and incongruently, with respect to the frame. From the perspective that proprioceptive dis- 
equilibrium heightens the observer's reliance on vision, congruent and incongruent roll should both lead to the same large RFI. However, Witkin and Asch (1948) reported that observers who were rolled incongruently with the visual frame experienced a larger RFI than did those who were rolled congruently. However, the mean difference in observers' judgments was small and not statistically significant. Furthermore, the observers were sitting in a chair, and the frame was viewed in an otherwise dark room; these two conditions have not been studied systematically. Importantly, sitting in a chair limits the corrective actions an observer can make in order to maintain an upright posture and therefore may limit access to the relevant proprioceptive information. Similarly, viewing the frame in the dark limits the use of other visual references, such as a visible horizon, that might otherwise be used to calibrate a sense of upright (Bressan, Garlaschelli, $\&$ Barracano, 2003). To examine the roles played by these other factors, we tested (1) observer roll that was congruent and incongruent with the tilt of the visual frame, (2) seated observers (as in Witkin \& Asch, 1948, and Asch \& Witkin, 1948b) and standing observers (as in Asch \& Witkin, 1948a), and (3) dark viewing (only the rod and frame were illuminated, as in Nelson \& Prinzmetal, 2003; Witkin \& Asch, 1948) and light viewing (as in Shimamura \& Prinzmetal, 1999).

\section{Method}

Participants. Eighteen individuals (10 females and 8 males, 18-35 years old) at the University of British Columbia participated voluntarily in two sessions. Five were undergraduates recruited from the human subject pool in the Department of Psychology, and they received extra course credit. Thirteen graduate students and postdoctoral fellows were offered refreshments for their participation. All of the participants had normal or corrected-to-normal vision and none reported visual or proprioceptive disorders.

The 13 graduate students and postdoctoral fellows participated in both a light and a dark session. However, the five undergraduates who were initially tested with the room lights on were unavailable for a second session. Therefore, 18 observers participated in a first testing session in the light and 13 of those participated in the second session in the dark, where only the rod and frame were illuminated.

Apparatus. A rod and frame box made of translucent white Plexiglas $(57 \mathrm{~cm}$ long $\times 31 \mathrm{~cm}$ high $\times 31 \mathrm{~cm}$ wide $)$ was used to induce the RFI, as illustrated in Figure 1. During testing, the rod could be rotated $360^{\circ}$, but the square frame was fixed at an orientation of $+20^{\circ}$ relative to horizontal. A protractor, displayed on a disc mounted on the rear of the box, and visible only to the experimenter, indicated the rod's deviation from upright $\left(0^{\circ}\right)$. The elevation of the box was adjusted so that it was at eye level when the observers were seated or standing. The position of the box was adjusted for each observer so that approximately $5 \mathrm{~cm}$ separated the eyes of the observer from the front edge of the box. The observers were supported either on a level floor (for the no-roll and no-pitch conditions) or on a $125-\mathrm{cm}^{2}$ wooden platform that was slanted $20^{\circ}$, relative to the floor. This platform could be rotated into positions marked on the floor in order to create active conditions for roll only, pitch only, or combined pitch and roll. A chair was bolted onto the platform for the passive viewing conditions. The platform and chair could be rotated independently to produce passive conditions for roll only, pitch only, or combined pitch and roll. We note that although pitch and roll were not fully mechanically independent in this apparatus, the apparatus did permit variation along each of these two dimensions. The partici-

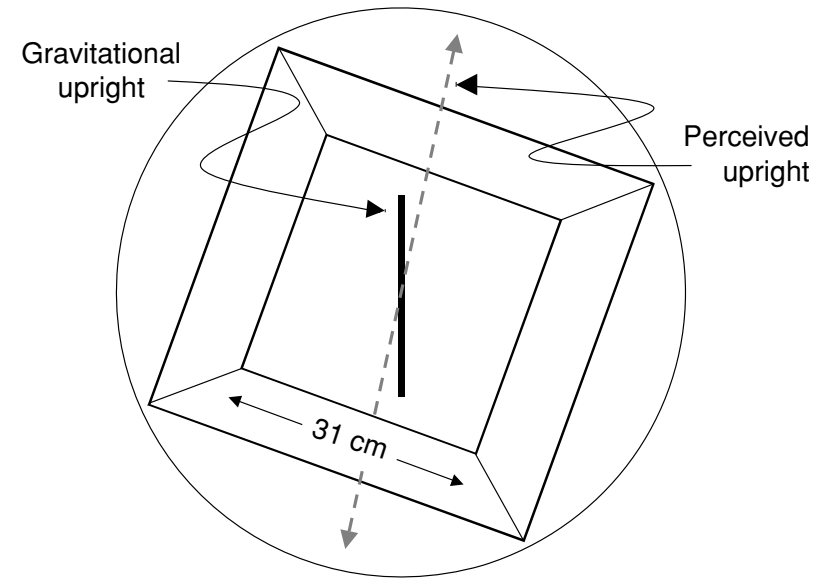

Figure 1. Participant's eye view of the rod and frame apparatus. The frame is rolled $+20^{\circ}$ to create the rod and frame illusion. When the rod is vertical with respect to gravity $\left(0^{\circ}\right.$, solid line $)$, it appears tilted to observers because their perception of upright errs in the direction of the tilted frame (dashed line).

pants wore a patch over one eye, so that all adjustments were made with their preferred eye.

Procedure. The participants peered inside the box and instructed the experimenter to rotate the rod until it was "vertical," defined as "in line with the pull of gravity." The participants were given a demonstration of how the rod could be rotated before testing began; testing did not begin until all questions regarding the procedure had been answered. The experimenter was unable to see the rod inside the box and could only see the protractor mounted on the rotating disc. The experimenter looked away from the box each time a participant asked for the disc to be rotated. The participants were given up to $1 \mathrm{~min}$ to refine their adjustments on each trial. The initial position of the rod alternated from trial to trial, beginning initially to the far right or far left of vertical.

The participants were first tested with the room lights on. Because there were $5 \mathrm{~cm}$ between their eyes and the front edge of the box, there was a possibility for peripheral vision of the room to play a role. The order of postural conditions was randomly determined. In the active condition, the participants stood on the platform. The experimenter, who stood at the other end of the box in order to move the rod according to the instructions of the participant, was able to make sure that the participant's body and head maintained an upright position and that his or her head did not move away from the box at any time. In the passive condition, the participants were seated in a chair that included a headrest to ensure that the head was kept in consistent orientation with the trunk.

The testing session in the dark occurred on a separate day. In this condition, a small bulb illuminated the interior of the rod and frame box to ensure that the rod and frame were as visible as they were in the lights-on condition. Each participant adjusted the rod a total of 12 times in each session ( 3 roll $\times 2$ pitch $\times 2$ postural conditions $)$.

\section{Results}

The mean settings (degrees of tilt from vertical) are shown in Table 1. These data were examined by ANOVA involving the factors of observer roll (none, congruent, incongruent), observer pitch (none, pitched), postural balance (active, passive), and lighting (light, dark).

Figure 2 illustrates the main findings. First, there was a significant RFI in both the active and passive condi- 
Table 1

Mean Rod Settings (in Degrees From Vertical) in Experiment 1

\begin{tabular}{|c|c|c|c|c|c|c|}
\hline \multirow[b]{3}{*}{ Pitch } & \multicolumn{6}{|c|}{ Observer Roll } \\
\hline & \multicolumn{2}{|c|}{ None } & \multicolumn{2}{|c|}{ Congruent } & \multicolumn{2}{|c|}{ Incongruent } \\
\hline & $M$ & $\overline{S E}$ & $M$ & $S E$ & $M$ & $S E$ \\
\hline \multicolumn{7}{|c|}{ Active Observers - Light $(n=18)$} \\
\hline None & 2.03 & 0.84 & 2.67 & 1.15 & 1.75 & 1.21 \\
\hline Backward & 1.58 & 0.95 & 2.64 & 1.10 & 1.92 & 0.94 \\
\hline \multicolumn{7}{|c|}{ Active Observers-Dark $(n=13)$} \\
\hline None & 3.19 & 0.81 & 3.54 & 0.76 & 3.08 & 0.92 \\
\hline Backward & 3.04 & 1.00 & 3.08 & 0.91 & 2.50 & 1.14 \\
\hline \multicolumn{7}{|c|}{ Passive Observers-Light $(n=18)$} \\
\hline None & 3.61 & 0.84 & 5.39 & 0.92 & 0.89 & 1.09 \\
\hline Backward & 1.11 & 0.98 & 4.98 & 1.24 & 0.31 & 0.84 \\
\hline \multicolumn{7}{|c|}{ Passive Observers-Dark $(n=13)$} \\
\hline None & 2.92 & 1.10 & 7.81 & 1.00 & 4.15 & 1.07 \\
\hline Backward & 3.62 & 0.86 & 5.77 & 1.19 & 3.08 & 0.95 \\
\hline
\end{tabular}

tions: Participants were never able to adjust the rod to $0^{\circ}$ of orientation in the context of the tilted visual frame (all $p$ values $<.01)$. Second, manipulations of observer roll and pitch each had an influence on the RFI for passive observers. Congruent observer roll increased RFI, whereas incongruent roll decreased the illusion, when compared with the no-roll condition. Observer pitch, on the other hand, decreased the RFI. Third, the influences of observer roll and pitch were independent, meaning that the effects of congruent and incongruent roll were the same, regardless of whether the observer was pitched or not. Fourth, although observers in the active condition were subject to the RFI, rod settings were not influenced significantly by variations in the roll or pitch of the platform. Fifth, whether the room lights were on or off had little influence on these findings. A single exception concerned the incongruent roll condition for passive observers in the light. Under these conditions, incongruent roll resulted in an RFI that was even smaller than in the no-roll baseline con- dition. Otherwise, incongruent roll resulted in essentially the same illusion as did the no-roll condition.

These conclusions were supported by ANOVA. A preliminary analysis of the 13 participants who were tested both in the light and in the dark revealed no significant main effect of light $\left[F(1,12)<1, M S_{\mathrm{e}}=117.282\right]$ and no interactions of light $\times$ activity $\left[F(1,12)<1, M S_{\mathrm{e}}=\right.$ $34.756]$, light $\times$ pitch $\left[F(1,12)<1, M S_{\mathrm{e}}=3.548\right]$, light $\times$ roll $\times$ pitch $\left[F(2,24)=2.66, M S_{\mathrm{e}}=6.346, p>.05\right]$, or light $\times$ activity $\times$ roll $\times$ pitch $\left[F(2,24)=1.99, M S_{\mathrm{e}}=\right.$ $5.267, p>.15]$. The only significant interactions involving light were light $\times$ roll $\left[F(2,24)=4.786, M S_{\mathrm{e}}=4.704\right.$, $p<.02]$ and light $\times$ activity $\times$ roll $[F(2,24)=5.52, p<$ $.01]$, which could both be attributed to a decreased illusion in the incongruent roll condition, but only when the participants were tested under passive postural control in the light. Under these conditions, the RFI was significantly smaller than it was in the no-roll control conditions $\left[F(1,24)=9.46, M S_{\mathrm{e}}=6.346, p<.01\right]$.

In order to maximize the statistical power for the consideration of observer pitch and roll, the remaining analyses were conducted on the data from all 18 observers who were tested in the light. This ANOVA revealed significant interactions of activity $\times$ roll $[F(2,34)=6.31$, $\left.M S_{\mathrm{e}}=9.462, p<.01\right]$ and activity $\times$ pitch $[F(1,17)=$ 4.64, $\left.M S_{\mathrm{e}}=3.126, p<.05\right]$, but no three-way interaction of activity $\times$ roll $\times$ pitch $\left[F(2,34)<1, M S_{\mathrm{e}}=6.678\right]$. Examination of these interactions indicated that the influences of observer roll and pitch were significant in the passive postural condition but not in the active condition (all $p \mathrm{~s}>.20$ ). Simple effects ANOVA in the passive condition revealed significant main effects of observer roll $\left[F(2,34)=19.47, M S_{\mathrm{e}}=9.462, p<.01\right]$ and observer pitch $\left[F(1,17)=11.39, M S_{\mathrm{e}}=3.126, p<.01\right]$, but no interaction of roll $\times$ pitch $[F(2,34)=1.66, p>.20]$. The main effect of roll was subdivided further into a significantly larger illusion for congruent roll than for no roll $\left[F(1,34)=8.64, M S_{\mathrm{e}}=9.462, p<.01\right]$ and a margin-

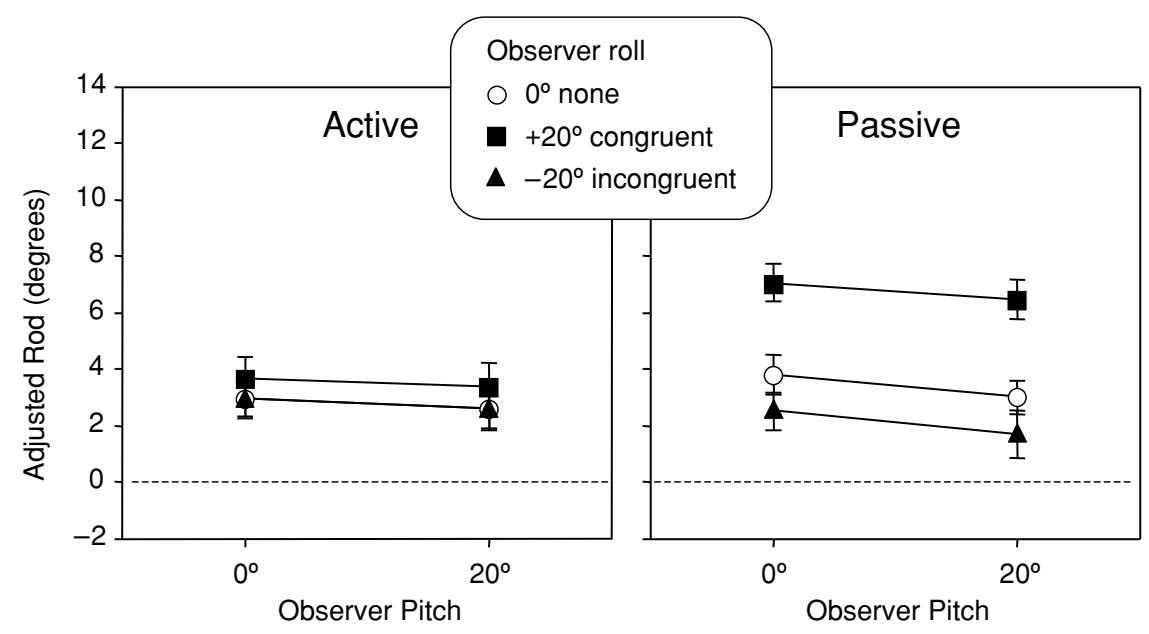

Figure 2. Mean rod and frame illusion in Experiment 1: Influences of pitch and roll of the floor in active and passive observers. Error bars indicate \pm 1 SEM. 
ally smaller illusion for incongruent roll than for no roll $\left[F(1,34)=2.889, M S_{\mathrm{e}}=9.462, p<.10\right]$.

\section{Discussion}

The main finding was that observer pitch and roll had independent influences on the RFI when observers were seated. The absence of a statistical interaction under these conditions is similar to the results of Nelson and Prinzmetal (2003), who found that pitch and roll of the visual environment contributed independently to observers' estimates of upright, even when observers were stationed on a flat surface. Together with the present finding, this suggests that separate mechanisms are at play in the perception of these two dimensions of space, regardless of whether they are perceived via visual, vestibular, or proprioceptive inputs.

Experiment 1 also contributed data to unresolved issues regarding the RFI. First, on the question of the congruent versus incongruent observer roll, the data are clear in showing that the largest illusion occurs when observers are oriented congruently with the frame. When observers are rolled incongruently, the RFI is similar to that measured when observers are not rolled at all. One explanation is that the congruent roll condition is most similar to the conditions of everyday viewing, in which the visual cues in the environment agree with the proprioceptive cues regarding upright; it is the reliance on visual cues under these everyday circumstances that contributes to the large illusion in the congruent experimental conditions. In contrast, when observers are rolled incongruently with the frame, the discrepancy between these two sources of information prompts observers to consult their vestibular and proprioceptive signals more carefully, permitting compensation for the tilted visual frame.

Support for this interpretation can be found in the interactions observed between light, postural activity, and observer roll. Recall that when observers were tested in the light, the incongruent roll condition resulted in a smaller RFI than when they were tested in the dark. This is consistent with observers using proprioception to compensate for the RFI normally experienced under observer roll. These signals would have greater support in the light condition, in which the rest of the testing room was partially visible and could be used to calibrate these cues, than in the dark condition, in which only the rod and frame were visible.

Additional support for the idea that observers were weighting their visual and proprioceptive cues differentially comes from the small but significant reduction in the RFI for pitched observers. When seated observers adjusted the rod, they experienced a smaller visual tilt illusion than when they were sitting upright. Pitching a seated person backward (or forward) in a chair gives him or her additional points of somatosensory contact (back and legs), possibly allowing the individual to read his or her body position better (including roll) than when the individual is seated upright.

The second issue concerns ambient visual lighting. For the most part, whether or not observers were able to see the room through peripheral vision had no effect on the RFI. This means that the stimuli considered "relevant" to the observer are restricted to the rod and frame apparatus. The one exception to this rule, already mentioned, concerns passively seated observers who are rolled incongruently with respect to the tilted frame. In this situation, it appears that peripheral vision helped observers compensate to some extent for the roll of the frame by allowing them to consult their proprioceptive signals relative to their ambient visual surroundings.

A final issue concerns seated as opposed to standing observers. The results showed that observers who were actively maintaining their balance experienced a robust RFI. However, their illusion was not influenced by the pitch and roll of the environment. In contrast, when observers were seated, variations in observer orientation had a large and significant influence. There are at least two explanations for this difference. First, active observers may have access to more accurate proprioceptive information than do passive observers, simply by virtue of the fact that they must use this information to maintain their balance. If so, then the reduced effects of observer pitch and roll for active observers are consistent with the compensation account already described: Proprioception is weighted more heavily when it is also being used for the purpose of standing upright. An alternative account is that the heads of active observers remained upright with respect to gravity, whereas the heads of passive observers were rolled. This postural adjustment by the active observers may have allowed them to experience the rod and frame in a way more similar to the way it is experienced by observers who are supported on a horizontal surface. We consider this issue in the next experiments.

\section{EXPERIMENT 2}

In Experiment 1, seated observers were influenced by the pitch and roll of the floor, but standing observers were not. Yet these conditions differed not only in whether participants made active postural adjustments; the heads of standing observers were upright, whereas those of seated observers were rolled. This may be important, because head orientation strongly influences the vestibular sense. Body balance is dependent, to a large extent, on the semicircular canals and otolith organs of the inner ear (Wilson \& Melvill Jones, 1979). Perhaps the sensitivity to pitch and roll in seated observers occurred because their vestibular sense of gravity was disrupted. If so, then we should be able to remove the proprioceptive influences on the RFI by allowing seated observers to hold their heads upright while the chair in which they are seated is pitched and rolled. Alternatively, if the vestibular system is not the principle sensory mechanism being disrupted by head and body roll, we should obtain the same results as in Experiment 1 (passive condition) even when the head is held upright.

\section{Method}

Twelve individuals ( 6 females and 6 males, $18-35$ years old) participated in a procedure identical to that used in the passive condi- 
tion (light viewing) in Experiment 1, with the exception that the headrest was removed from the chair and all of the participants were instructed to keep their heads upright during the session. The experimenter, standing at the other end of the box, was able to make sure that the participant's head was maintained in an upright position using a horizontal reference line on the wall behind the participant.

\section{Results}

Figure 3 shows two main findings. First, there was a significant RFI in each condition of the experiment (all $p$ values $<.01$ ) that was similar in magnitude to the RFI measured in the active condition of Experiment $1(F<1)$. Second, manipulations of observer roll and pitch on seated observers no longer had any influence on the RFI (all $F$ values for main effects and interactions $<1$ ).

\section{Discussion}

The results suggest that the proprioceptive influences on the RFI observed in Experiment 1 occurred because the vestibular system was disrupted by rolling the head. When observers were able to hold their heads upright in the present experiment, there was still a significant RFI, but it was no longer influenced by the position of the observer's body relative to the pull of gravity. In other words, the results were now indistinguishable from those in the active condition of Experiment 1, in which observers were standing upright on a pitched and rolled platform.

\section{EXPERIMENT 3}

As a final step, we asked whether disrupting the vestibular system alone was sufficient to reinstate the proprioceptive effects observed in the passive condition of Experiment 1. We tested this by measuring the RFI while participants were standing upright on the pitched and rolled platform, but with the requirement that they roll their heads $20^{\circ}$ to one side, so their heads would be congruent with the visual frame. If vestibular disruption was responsible for the proprioceptive influences on the RFI,

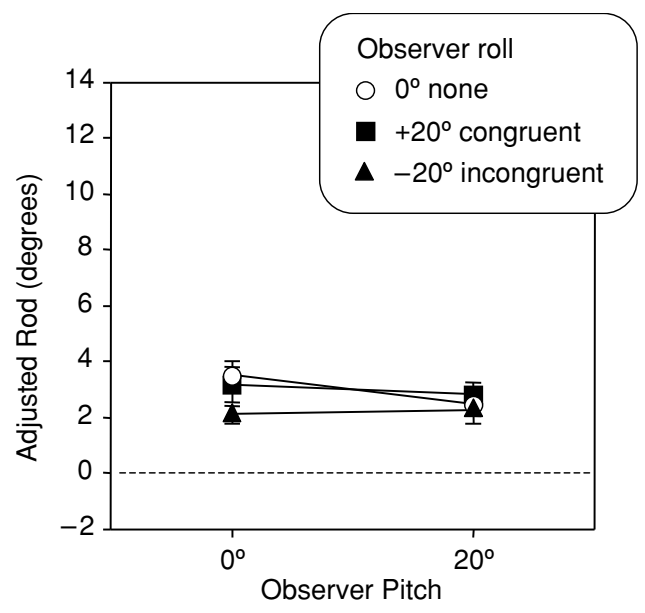

Figure 3. Mean rod and frame illusion in Experiment 2: Passively pitched and rolled observers with vertical head orientation. Error bars indicate $\pm 1 S E M$. then this manipulation should reinstate them. Alternatively, if the combination of body and head roll was necessary to cause the proprioceptive influences in Experiment 1 , then a simple roll of the head should not reveal any proprioceptive influences.

\section{Method}

The 12 participants from Experiment 2 were tested in a procedure identical to that used in the active condition (light viewing) in Experiment 1 , with the exception that they aligned their heads with the tilt of the frame. The experimenter monitored the head position of all of the observers relative to a $20^{\circ}$ reference line on the wall directly behind the participants' heads.

\section{Results}

Figure 4 shows two main findings. First, there was a significant RFI in each condition of the experiment (all $p$ values $<.01$ ) that was much larger in magnitude than was the RFI measured in the active condition in Experiment 1 $[F(1,23)=24.60, p<.001]$. Second, manipulations of observer roll and pitch on seated observers had no measurable influence on the RFI (all $F$ values for main effects and interactions $<1.5, p>.24)$.

\section{Discussion}

The proprioceptive influences on the RFI observed in the passive condition of Experiment 1 appear to depend on both passive seating and a rolled head. As we saw in Experiment 3, simply rolling the observer's head so that it was congruent with the visual frame was not sufficient to invoke the proprioceptive influences on the RFI from the pitched and rolled platform that was supporting the participants. Experiment 2 showed that passive seating on a pitched and rolled chair was also not sufficient in itself to induce these effects. Only when the observer's head was rolled (to disrupt the vestibular sense of upright) and when the observer was seated in a chair that was pitched and rolled (to accentuate the proprioceptive inputs concerning the unusual environment) was the RFI influenced by both the pitch and roll of the floor supporting the observers.

\section{GENERAL DISCUSSION}

The consistent RFI that was measured in three experiments points to the large extent to which our perception of upright depends on vision. This reliance on vision becomes even stronger when the vestibular sense is disrupted by the act of rolling one's head to the side (as demonstrated in Experiment 3). In contrast, merely rolling one's body, without a corresponding roll of the head, does not result in a stronger-than-normal illusion (Experiment 2), presumably because the vestibular system can still be used to inform judgments about upright based on an upright head position. Finally, when both the vestibular system and the proprioceptive sense of upright are disturbed (Experiment 1 , passive condition), the visual illusion (RFI) becomes sensitive to proprioceptive signals regarding gravitational upright.

This pattern of data suggests a hierarchy of control signals with regard to the perception of upright. In this 


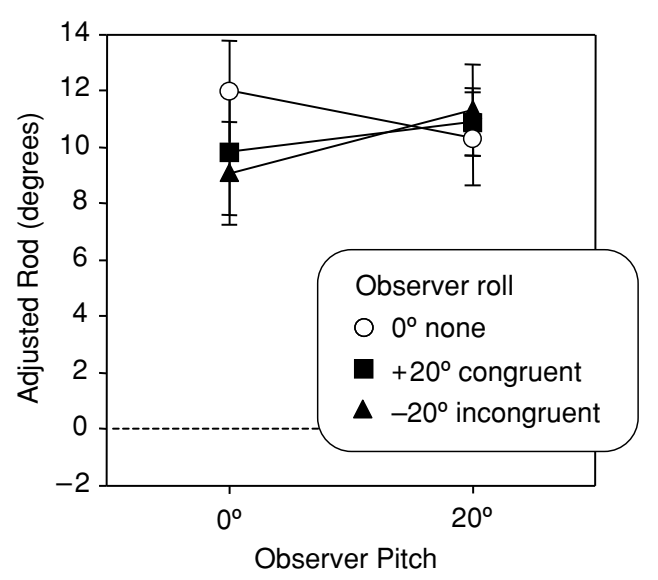

Figure 4. Mean rod and frame illusion in Experiment 3: Active observers with head orientation rolled $+20^{\circ}$. Error bars indicate \pm 1 SEM.

hierarchy, vision is the dominant sensory system, providing an illusion that is never completely overcome under any circumstance when the larger visual context is a tilted frame. The vestibular system has secondary status, in that it is permitted to modulate the visual input. This is presumably why the RFI is so large when the head is tilted and why it is reduced by the simple act of holding the head upright. Finally, the proprioceptive system does contribute to visual judgments of upright, but only if the vestibular sense has first become unreliable because of a head position that is less than vertical.

The finding of independent effects of observer roll and pitch in seated observers (Experiment 1) bears a superficial resemblance to the report that the pitch and roll of viewed surfaces contribute separately to the RFI (Nelson \& Prinzmetal, 2003). A promising lead for future studies of this independence is the observation that the vestibular system (semicircular canals and otolith organs) is organized to permit independent registration of the pitch and roll of the head (Wilson \& Melvill Jones, 1979). Along the same lines, recordings from muscles of the legs and trunk show separate responses to perturbations along the pitch and roll axes of rotation (Carpenter, Allum, \& Honegger, 1999). Moreover, vestibular neurons signaling pitch and roll maintain their distinctiveness in the visuomotor areas of the brain to which they project (Carpenter et al., 1999). It is therefore possible that functionally separate mechanisms for the visual perception of pitch and roll may have come about, in an evolutionary sense, from their connection to the more primitive vestibular and proprioceptive systems from which they emerged. If so, this suggests that future research will continue to find independent mechanisms for the perception of pitch and roll, regardless of the sensory system being studied.

\section{REFERENCES}

Asch, S. E., \& WitKIn, H. A. (1948a). Studies in space orientation: I. Perception of the upright with displaced visual fields. Journal of Experimental Psychology, 38, 325-337.

Asch, S. E., \& WitKIn, H. A. (1948b). Studies in space orientation: II. Perception of the upright with displaced visual fields and with body tilted. Journal of Experimental Psychology, 38, 455-477.

Bressan, P., Garlaschelli, L., \& Barracano, M. (2003). Antigravity hills are visual illusions. Psychological Science, 14, 441-449.

Carpenter, M. G., Allum, J. H. J., \& Honegger, F. (1999). Directional sensitivity of stretch reflexes and balance corrections for normal subjects in the roll and pitch planes. Experimental Brain Research, 129, 93-113.

Howard, I. P. (1982). Human visual orientation. New York: Wiley.

HowARD, I. P. (1986). The perception of posture, self motion, and the visual vertical. In K. R. Boff, L. Kaufman, \& J. P. Thomas (Eds.), Handbook of perception and human performance: Vol. 1. Sensory processes and perception (pp. 18.1-18.62). New York: Wiley.

Nelson, R., \& Prinzmetal, W. (2003). On the relation between roll and pitch. Visual Cognition, 10, 715-728.

Prinzmetal, W., \& BecK, D. M. (2001). The tilt-consistency theory of visual illusions. Journal of Experimental Psychology: Human Perception \& Performance, 27, 206-217.

Shimamura, A. P., \& Prinzmetal, W. (1999). The Mystery Spot illusion and its relation to other visual illusions. Psychological Science, 10, 501-507.

Wilson, V. J., \& Melvill Jones, G. (1979). Mammalian vestibular physiology. New York: Plenum.

WITKIN, H. A., \& Asch, S. E. (1948). Studies in space orientation: IV. Further experiments on perception of the upright with displaced visual fields. Journal of Experimental Psychology, 38, 762-782.

Young, L. R. (1984). Perception of the body in space: Mechanisms. In J. M. Brookhart \& V. B. Mountcastle (Section Eds.) \& I. Darian-Smith (Vol. Ed.), Handbook of physiology: Section 1. The nervous system: Vol. 3. Sensory processes (pp. 1023-1066). Bethesda, MD: American Physiological Society.

(Manuscript received October 3, 2004; revision accepted for publication June 12, 2005.) 